\title{
Global Exponential Stability of Positive Almost Periodic Solutions for a Delay Logarithmic Population Model
}

\author{
Wei Chen ${ }^{1}$ and Wentao Wang ${ }^{2}$ \\ ${ }^{1}$ School of Mathematics and Information, Shanghai Lixin University of Commerce, Shanghai 201620, China \\ ${ }^{2}$ College of Mathematics, Physics and Information Engineering, Jiaxing University, Jiaxing, Zhejiang 314001, China \\ Correspondence should be addressed to Wentao Wang; wwt@mail.zjxu.edu.cn
}

Received 4 October 2013; Accepted 17 April 2014; Published 13 May 2014

Academic Editor: Vu Ngoc Phat

Copyright (c) 2014 W. Chen and W. Wang. This is an open access article distributed under the Creative Commons Attribution License, which permits unrestricted use, distribution, and reproduction in any medium, provided the original work is properly cited.

This paper is concerned with a delay logarithmic population model. Under proper conditions, we employ a novel proof to establish a criterion on guaranteeing the existence and global exponential stability of positive almost periodic solutions for the model. Moreover, an example and its numerical simulation are given to illustrate the main results.

\section{Introduction}

In the classic study of population dynamics, the dynamical analysis of logarithmic population model has attracted a great attention of many mathematicians and biologists in recent years. It is well known that the environment is varying periodically with time in many realistic systems and the parameters of the system usually change along with time periodically. So it is reasonable to study periodic solution for logarithmic population model and its modified model with periodic coefficients. There exist some results on the existence of periodic solution for the model; see, for example, [1-8]. Compared with periodic effects, almost periodic effects are more frequent (see [9-12]). Hence, it is of great importance to consider the dynamical behaviors of logarithmic population model with almost periodically varying coefficient. Recently, by utilizing the continuation theorem and contraction mapping principle, some criteria have been established to prove the existence and local exponential stability of positive almost periodic solutions for delay logarithmic population model and its generalized modification in the literature; see [1317]. However, to the best of our knowledge, there is no literature considering the existence and global exponential stability of positive almost periodic solutions problem for delay logarithmic population model.
Inspired by the above discussions, in this paper, we consider the following delay logarithmic population equation:

$$
\begin{array}{r}
x^{\prime}(t)=x(t)\left[\gamma(t)-a_{1}(t) \ln x(t)-a_{2}(t) \ln x(t-\tau(t))\right], \\
t \geq 0,
\end{array}
$$

which was proposed by Gopalsamy [18] to describe a model of single species population. Here $x$ is the size of population, $\gamma(t)$ is the growth rate while there are plenty of resources and there is no intraspecific competition for these resources, $a_{1}(t)$ is the measure of the competition among the individuals, $a_{2}(t)$ is added to generalize the model with the same interpretation of competitive effects, and $\tau(t)$ is a maturation delay in the sense that competition involves adults who have matured by an age of $\tau(t)$ units.

A primary purpose of this paper is to consider the existence and global exponential stability of positive almost periodic solutions of (1). A new approach will be developed to obtain a delay-independent condition for the global exponential stability of the positive almost periodic solutions of (1). 
For convenience, we introduce some notations. Given a bounded continuous function $g$ defined on $\mathbb{R}$, let $g^{+}$and $g^{-}$ be defined as

$$
g^{+}=\sup _{t \in \mathbb{R}} g(t), \quad g^{-}=\inf _{t \in \mathbb{R}} g(t) .
$$

It will be assumed that $\gamma, a_{1}, a_{2}, \tau: \mathbb{R} \rightarrow[0,+\infty)$ are continuous almost periodic functions. Let $\mathbb{R}_{+}$denote the nonnegative real number space, let $C=C\left(\left[-\tau^{+}, 0\right], \mathbb{R}\right)$ be the continuous functions space equipped with the usual supremun norm $\|\cdot\|$, and let $C_{+}=C\left(\left[-\tau^{+}, 0\right], \mathbb{R}_{+}\right)$.

Due to the biological interpretation of model (1), only positive solutions are meaningful and therefore admissible. Thus we just consider admissible initial conditions

$$
x(t)=\varphi(t), \quad t \in\left[-\tau^{+}, 0\right], \varphi \in C_{+} .
$$

Let $x(t)=e^{y(t)}$; then (1) and admissible initial conditions (3) can be rewritten in the form

$$
\begin{gathered}
y^{\prime}(t)=\gamma(t)-a_{1}(t) y(t)-a_{2}(t) y(t-\tau(t)), \quad t \geq 0 \\
y(t)=\phi(t), \quad t \in\left[-\tau^{+}, 0\right], \phi \in C
\end{gathered}
$$

Obviously, model (1) has a unique positive almost periodic solution which is globally exponentially stable if and only if system (4) has a unique almost periodic solution which is globally exponentially stable.

\section{Preliminary Results}

In this section, some lemmas and definitions will be presented, which are of great significance in proving our main results in Section 3.

Definition 1 (see $[9,10]$ ). Let $u(t): \mathbb{R} \rightarrow \mathbb{R}$ be continuous in $t . u(t)$ is said to be almost periodic on $\mathbb{R}$ if, for any $\varepsilon>0$, the set $T(u, \varepsilon)=\{\delta:|u(t+\delta)-u(t)|<\varepsilon$ for all $t \in \mathbb{R}\}$ is relatively dense; that is, for any $\varepsilon>0$, it is possible to find a real number $l=l(\varepsilon)>0$, and for any interval with length $l(\varepsilon)$, there exists a number $\delta=\delta(\varepsilon)$ in this interval such that $|u(t+\delta)-u(t)|<\varepsilon$, for all $t \in \mathbb{R}$.

From the theory of almost periodic functions in $[9,10]$, it follows that, for any $\epsilon>0$, it is possible to find a real number $l=l(\epsilon)>0$, and for any interval with length $l(\epsilon)$, there exists a number $\delta=\delta(\epsilon)$ in this interval such that

$$
\begin{array}{lc}
|\gamma(t+\delta)-\gamma(t)|<\epsilon, & \left|a_{1}(t+\delta)-a_{1}(t)\right|<\epsilon, \\
\left|a_{2}(t+\delta)-a_{2}(t)\right|<\epsilon, & |\tau(t+\delta)-\tau(t)|<\epsilon,
\end{array}
$$

for all $t \in \mathbb{R}$.

Lemma 2. Suppose that $a_{1}^{-}-a_{2}^{+}>0$ and $y(t)$ is a solution of (4) with initial conditions satisfying (5) and the following condition:

$$
|\phi(t)|<\frac{\gamma^{+}+1}{a_{1}^{-}-a_{2}^{+}}
$$

Then, for t in the interval of existence

$$
|y(t)|<\frac{\gamma^{+}+1}{a_{1}^{-}-a_{2}^{+}}
$$

Proof. Assume, by way of contradiction, that (8) does not hold. Then suppose that there exists $t^{*}>0$ such that

$$
\left|y\left(t^{*}\right)\right|=\frac{\gamma^{+}+1}{a_{1}^{-}-a_{2}^{+}}, \quad|y(t)|<\frac{\gamma^{+}+1}{a_{1}^{-}-a_{2}^{+}}, \quad \forall t \in\left[-\tau^{+}, t^{*}\right) .
$$

Calculating the upper left-hand derivative of $|y(t)|$, it follows from (4) and (9) that

$$
\begin{aligned}
0 & \leq D^{-}\left(\left|y\left(t^{*}\right)\right|\right) \\
& \leq \gamma\left(t^{*}\right)-a_{1}\left(t^{*}\right)\left|y\left(t^{*}\right)\right|+a_{2}\left(t^{*}\right)\left|y\left(t^{*}-\tau\left(t^{*}\right)\right)\right| \\
& \leq \gamma^{+}-a_{1}^{-}\left|y\left(t^{*}\right)\right|+a_{2}^{+}\left|y\left(t^{*}\right)\right| \\
& =\gamma^{+}-\left(a_{1}^{-}-a_{2}^{+}\right) \frac{\gamma^{+}+1}{a_{1}^{-}-a_{2}^{+}} \\
& <0,
\end{aligned}
$$

which is contradictory and shows that (8) holds.

Remark 3. By virtue of the boundedness of this solution, from the theory of functional differential equations in [19], it follows that the solution of system (4) with initial conditions (7) can be defined on $[0,+\infty)$.

Lemma 4. Let $a_{1}^{-}-a_{2}^{+}>0$ hold. Assume that $y(t)$ is a solution of (4) with initial condition $\phi$ such that (7) is satisfied. Then for any $\epsilon>0$, there exists $l=l(\epsilon)>0$, such that every interval $[\alpha, \alpha+l]$ contains at least one number $\delta$ for which there exists $N>0$ which satisfies

$$
|y(t+\delta)-y(t)| \leq \epsilon, \quad \forall t>N
$$

Proof. Define a continuous function $\Gamma(\mu)$ by setting

$$
\Gamma(\mu)=\mu-a_{1}^{-}+a_{2}^{+} e^{\mu \tau^{+}}, \quad \mu \in[0,1] .
$$

Then, we have

$$
\Gamma(0)=-a_{1}^{-}+a_{2}^{+}<0,
$$

which implies that there exist two constants $\eta>0$ and $\lambda \epsilon$ $(0,1]$ such that

$$
\Gamma(\lambda)=\lambda-a_{1}^{-}+a_{2}^{+} e^{\lambda \tau^{+}}<-\eta .
$$

For $t \in\left(-\infty,-\tau^{+}\right]$, we add the definition of $y(t)$ with $y(t) \equiv$ $y\left(-\tau^{+}\right)$. Set

$$
\begin{aligned}
\epsilon(\delta, t)= & -\left[a_{1}(t+\delta)-a_{1}(t)\right] y(t+\delta) \\
& -\left[a_{2}(t+\delta)-a_{2}(t)\right] y(t+\delta-\tau(t+\delta)) \\
& -a_{2}(t)[y(t+\delta-\tau(t+\delta))-y(t+\delta-\tau(t))] \\
& +\gamma(t+\delta)-\gamma(t), \quad t \in R .
\end{aligned}
$$


By Lemma 2, the solution $y(t)$ is bounded and

$$
|y(t)|<\frac{\gamma^{+}+1}{a_{1}^{-}-a_{2}^{+}}, \quad \forall t \in \mathbb{R},
$$

which implies that the right side of (4) is also bounded, and $y^{\prime}(t)$ is a bounded function on $\left[-\tau^{+},+\infty\right)$. Thus, in view of the fact that $y(t) \equiv y\left(-\tau^{+}\right)$for $t \in\left(-\infty,-\tau^{+}\right]$, we obtain that $y(t)$ is uniformly continuous on $\mathbb{R}$. From (6), for any $\epsilon>0$, there exists $l=l(\epsilon)>0$, such that every interval $[\alpha, \alpha+l]$, $\alpha \in \mathbb{R}$, contains a $\delta$ for which

$$
|\epsilon(\delta, t)| \leq \frac{1}{2} \eta \epsilon, \quad \forall t \in \mathbb{R}
$$

Recall that $\eta$ is the same as the one mentioned in (14).

Let $N_{0} \geq \max \{0,-\delta\}$. For $t \in \mathbb{R}$, denote

$$
u(t)=y(t+\delta)-y(t) .
$$

Then, for all $t \geq N_{0}$, we get

$$
\begin{aligned}
\frac{d u(t)}{d t}= & \gamma(t+\delta)-a_{1}(t+\delta) y(t+\delta) \\
& -a_{2}(t+\delta) y(t+\delta-\tau(t+\delta)) \\
& -\left[\gamma(t)-a_{1}(t) y(t)-a_{2}(t) y(t-\tau(t))\right] \\
= & -a_{1}(t) u(t)-a_{2}(t) u(t-\tau(t))+\epsilon(\delta, t) .
\end{aligned}
$$

Calculating the left upper derivative of $e^{\lambda t}|u(t)|$ yields

$$
\begin{aligned}
& D^{-}\left(e^{\lambda t}|u(t)|\right) \\
& \leq \lambda e^{\lambda t}|u(t)| \\
& \quad+e^{\lambda t}\left\{-a_{1}(t)|u(t)|+\left|-a_{2}(t) u(t-\tau(t))+\epsilon(\delta, t)\right|\right\} \\
& \leq\left(\lambda-a_{1}(t)\right) e^{\lambda t}|u(t)|+a_{2}(t) e^{\lambda t}|u(t-\tau(t))| \\
& \quad+e^{\lambda t}|\epsilon(\delta, t)| .
\end{aligned}
$$

Let

$$
U(t)=\sup _{-\infty<s \leq t}\left\{e^{\lambda s}|u(s)|\right\}
$$

It is obvious that $e^{\lambda t}|u(t)| \leq U(t)$ and that $U(t)$ is nondecreasing.

Now, we distinguish two cases to finish the proof since $e^{\lambda t}|u(t)|<U(t)$ and $e^{\lambda t}|u(t)|=U(t)$ are both possible.

Case 1. Consider the following:

$$
U(t)>e^{\lambda t}|u(t)| \quad \forall t \geq N_{0} .
$$

We claim that

$$
U(t) \equiv U\left(N_{0}\right) \text { is a constant } \forall t \geq N_{0} .
$$

Assume, by way of contradiction, that (23) does not hold. Then, there exists $t_{1}>N_{0}$ such that $U\left(t_{1}\right)>U\left(N_{0}\right)$. Since

$$
e^{\lambda t}|u(t)| \leq U\left(N_{0}\right) \quad \forall t \leq N_{0},
$$

there must exist $\beta \in\left(N_{0}, t_{1}\right)$ such that

$$
e^{\lambda \beta}|u(\beta)|=U\left(t_{1}\right) \geq U(\beta),
$$

which contradicts (22). This contradiction implies that (23) holds. It follows that there exists $t_{2}>N_{0}$ such that

$$
|u(t)| \leq e^{-\lambda t} U(t)=e^{-\lambda t} U\left(N_{0}\right)<\epsilon \quad \forall t \geq t_{2} .
$$

Case 2. There is a $t_{0}^{*} \geq N_{0}$ that $U\left(t_{0}^{*}\right)=e^{\lambda t_{0}^{*}}\left|u\left(t_{0}^{*}\right)\right|$. Then, in view of (14) and (20), we get

$$
\begin{aligned}
0 \leq & \left.D^{-}\left(e^{\lambda t}|u(t)|\right)\right|_{t=t_{0}^{*}} \\
\leq & \left(\lambda-a_{1}\left(t_{0}^{*}\right)\right) e^{\lambda t_{0}^{*}}\left|u\left(t_{0}^{*}\right)\right| \\
& +a_{2}\left(t_{0}^{*}\right) e^{\lambda \tau\left(t_{0}^{*}\right)} e^{\lambda\left(t_{0}^{*}-\tau\left(t_{0}^{*}\right)\right)}\left|u\left(t_{0}^{*}-\tau\left(t_{0}^{*}\right)\right)\right| \\
& +e^{\lambda t_{0}^{*}}\left|\epsilon\left(\delta, t_{0}^{*}\right)\right| \\
\leq & \left(\lambda-a_{1}\left(t_{0}^{*}\right)+a_{2}\left(t_{0}^{*}\right) e^{\lambda \tau\left(t_{0}^{*}\right)}\right) U\left(t_{0}^{*}\right)+e^{\lambda t_{0}^{*}}\left|\epsilon\left(\delta, t_{0}^{*}\right)\right| \\
\leq & \left(\lambda-a_{1}^{-}+a_{2}^{+} e^{\lambda \tau^{+}}\right) U\left(t_{0}^{*}\right)+\frac{1}{2} \eta \epsilon e^{\lambda t_{0}^{*}} \\
< & -\eta U\left(t_{0}^{*}\right)+\eta \epsilon e^{\lambda t_{0}^{*}},
\end{aligned}
$$

which yields that

$$
e^{\lambda t_{0}^{*}}\left|u\left(t_{0}^{*}\right)\right|=U\left(t_{0}^{*}\right)<\epsilon e^{\lambda t_{0}^{*}}, \quad\left|u\left(t_{0}^{*}\right)\right|<\epsilon .
$$

For any $t>t_{0}^{*}$, with the same approach as that in deriving of (28), we can show

$$
e^{\lambda t}|u(t)|<\epsilon e^{\lambda t}, \quad|u(t)|<\epsilon,
$$

if $U(t)=e^{\lambda t}|u(t)|$.

On the other hand, if $U(t)>e^{\lambda t}|u(t)|$ and $t>t_{0}^{*}$, we can choose $t_{0}^{*} \leq t_{3}<t$ such that

$$
U\left(t_{3}\right)=e^{\lambda t_{3}}\left|u\left(t_{3}\right)\right|, \quad U(s)>e^{\lambda s}|u(s)| \quad \forall s \in\left(t_{3}, t\right],
$$

which, together with (29), yields

$$
\left|u\left(t_{3}\right)\right|<\epsilon .
$$

With a similar argument as that in the proof of Case 1 , we can show that

$$
U(s) \equiv U\left(t_{3}\right) \text { is a constant } \forall s \in\left(t_{3}, t\right],
$$

which implies that

$$
|u(t)|<e^{-\lambda t} U(t)=e^{-\lambda t} U\left(t_{3}\right)=\left|u\left(t_{3}\right)\right| e^{-\lambda\left(t-t_{3}\right)}<\epsilon .
$$

In summary, there must exist $N>\max \left\{t_{0}^{*}, N_{0}, t_{2}\right\}$ such that $|u(t)| \leq \epsilon$ holds for all $t>N$. The proof of Lemma 4 is now complete. 


\section{Main Results}

In this section, we establish sufficient conditions on the existence and global exponential stability of almost periodic solutions of (4).

Theorem 5. Under the assumptions of Lemma 4, (4) has at least one almost periodic solution $y^{*}(t)$.

Proof. Let $v(t)$ be a solution of (4) with initial conditions satisfying the assumptions in Lemma 4 . We also add the definition of $v(t)$ with $v(t) \equiv v\left(-\tau^{+}\right)$for all $t \in\left(-\infty,-\tau^{+}\right]$. Set

$$
\begin{aligned}
\epsilon(k, t)= & -\left[a_{1}\left(t+t_{k}\right)-a_{1}(t)\right] v\left(t+t_{k}\right) \\
& -\left[a_{2}\left(t+t_{k}\right)-a_{2}(t)\right] v\left(t+t_{k}-\tau\left(t+t_{k}\right)\right) \\
& -a_{2}(t)\left[v\left(t+t_{k}-\tau\left(t+t_{k}\right)\right)-v\left(t+t_{k}-\tau(t)\right)\right] \\
& +\gamma\left(t+t_{k}\right)-\gamma(t), \quad t \in \mathbb{R},
\end{aligned}
$$

where $\left\{t_{k}\right\}$ is any sequence of real numbers. By Lemma 4 , the solution $v(t)$ is bounded and

$$
|v(t)|<\frac{\gamma^{+}+1}{a_{1}^{-}-a_{2}^{+}}, \quad \forall t \in \mathbb{R}
$$

which implies that the right side of (4) is also bounded, and $v^{\prime}(t)$ is a bounded function on $\left[-\tau^{+},+\infty\right)$. Thus, in view of the fact that $v(t) \equiv v\left(-\tau^{+}\right)$for $t \in\left(-\infty,-\tau^{+}\right]$, we obtain that $v(t)$ is uniformly continuous on $\mathbb{R}$. Then, from the almost periodicity of $\gamma, a_{1}, a_{2}$, and $\tau$, we can select a sequence $\left\{t_{k}\right\} \rightarrow+\infty$ such that

$$
\begin{aligned}
&\left|\gamma\left(t+t_{k}\right)-\gamma(t)\right| \leq \frac{1}{k},\left|a_{1}\left(t+t_{k}\right)-a_{1}(t)\right| \leq \frac{1}{k} \\
&\left|a_{2}\left(t+t_{k}\right)-a_{2}(t)\right| \leq \frac{1}{k},\left|\tau\left(t+t_{k}\right)-\tau(t)\right| \leq \frac{1}{k} \\
& \varepsilon|k, t| \leq \frac{1}{k} \\
& \forall t .
\end{aligned}
$$

Since $\left\{v\left(t+t_{k}\right)\right\}_{k=1}^{+\infty}$ is uniformly bounded and equiuniformly continuous, by Arzala-Ascoli lemma and diagonal selection principle, we can choose a subsequence $\left\{t_{k_{j}}\right\}$ of $\left\{t_{k}\right\}$, such that $v\left(t+t_{k_{j}}\right)$ (for convenience, we still denote by $v\left(t+t_{k}\right)$ ) uniformly converges to a continuous function $y^{*}(t)$ on any compact set of $\mathbb{R}$, and

$$
\left|y^{*}(t)\right| \leq \frac{\gamma^{+}+1}{a_{1}^{-}-a_{2}^{+}}, \quad \forall t \in \mathbb{R}
$$

In the sequel, we prove that $y^{*}(t)$ is a solution of (4). In fact, for any $t \geq 0$ and $\Delta t \in \mathbb{R}$, from (36), we have

$$
\begin{aligned}
& y^{*}(t+\Delta t)-y^{*}(t) \\
& =\lim _{k \rightarrow+\infty}\left[v\left(t+\Delta t+t_{k}\right)-v\left(t+t_{k}\right)\right] \\
& =\lim _{k \rightarrow+\infty} \int_{t}^{t+\Delta t}\left\{\gamma\left(\mu+t_{k}\right)-a_{1}\left(\mu+t_{k}\right) v\left(\mu+t_{k}\right)\right. \\
& \left.\quad-a_{2}\left(\mu+t_{k}\right) v\left(\mu+t_{k}-\tau\left(\mu+t_{k}\right)\right)\right\} d \mu \\
& =\lim _{k \rightarrow+\infty} \int_{t}^{t+\Delta t}\left\{\gamma(\mu)-a_{1}(\mu) v\left(\mu+t_{k}\right)\right. \\
& =\lim _{k \rightarrow+\infty} \int_{t}^{t+\Delta t}\left\{\gamma(\mu)-a_{1}(\mu) v\left(\mu+t_{k}\right)\right. \\
& \left.\quad-a_{2}(\mu) v\left(\mu+t_{k}-\tau(\mu)\right)\right\} d \mu \\
& +\lim _{k \rightarrow+\infty} \int_{t}^{t+\Delta t} \varepsilon(k, \mu) d \mu \\
& \left.\lim _{k \rightarrow+\infty} \int_{t}^{t+\Delta t}\left\{\gamma(\mu)-a_{1}(\mu) y^{*}(\mu)\right)+\varepsilon(k, \mu)\right\} d \mu \\
& \left.-a_{2}(\mu) y^{*}(\mu-\tau(\mu))\right\} d \mu,
\end{aligned}
$$

where $t+\Delta t \geq 0$. Consequently, (38) implies that

$$
\frac{d}{d t}\left\{y^{*}(t)\right\}=\gamma(t)-a_{1}(t) y^{*}(t)-a_{2}(t) y^{*}(t-\tau(t))
$$

Therefore, $y^{*}(t)$ is a solution of (4).

Then, we prove that $y^{*}(t)$ is an almost periodic solution of (4). From Lemma 4, for any $\varepsilon>0$, there exists $l=l(\varepsilon)>0$, such that every interval $[\alpha, \alpha+l]$ contains at least one number $\delta$ for which there exists $N>0$ which satisfies

$$
|v(t+\delta)-v(t)| \leq \varepsilon, \quad \forall t>N
$$

Then, for any fixed $s \in \mathbb{R}$, we can find a sufficient large positive integer $N_{1}>N$ such that for any $k>N_{1}$,

$$
s+t_{k}>N, \quad\left|v\left(s+t_{k}+\delta\right)-v\left(s+t_{k}\right)\right| \leq \varepsilon .
$$

Let $k \rightarrow+\infty$; we obtain

$$
\left|y^{*}(s+\delta)-y^{*}(s)\right| \leq \varepsilon,
$$

which implies that $y^{*}(t)$ is an almost periodic solution of (4). This completes the proof.

The following result implies that (4) has a unique almost periodic solution.

Theorem 6. Suppose that all conditions in Theorem 5 are satisfied. Then (4) has an almost periodic solution which is globally exponentially stable. 
Proof. Theorem 5 tells us that (4) has at least one almost periodic solution. Let $y^{*}(t)$ be one of them. We show that $y^{*}(t)$ is globally exponentially stable. Let $y(t)$ be an arbitrary solution of (4) and define $z(t)=y(t)-y^{*}(t)$, where $t \epsilon$ $\left[-\tau^{+},+\infty\right)$. Then

$$
z^{\prime}(t)=-a_{1}(t) z(t)-a_{2}(t) z(t-\tau(t)), \quad t \geq 0 .
$$

We consider the Lyapunov functional

$$
V(t)=|z(t)| e^{\lambda t} .
$$

Calculating the upper left derivative of $V(t)$ along the solution $z(t)$ of (43), we have

$$
\begin{aligned}
& D^{-}(V(t)) \\
& \leq \lambda|z(t)| e^{\lambda t}+e^{\lambda t}\left(-a_{1}(t)|z(t)|+a_{2}(t)|z(t-\tau(t))|\right) \\
& \leq\left(\lambda-a_{1}^{-}\right)|z(t)| e^{\lambda t}+a_{2}^{+} e^{\lambda \tau^{+}}|z(t-\tau(t))| e^{\lambda(t-\tau(t))} .
\end{aligned}
$$

We claim that

$$
\begin{aligned}
V(t) & =|z(t)| e^{\lambda t} \\
& <\max _{t \in\left[-\tau^{+}, 0\right]}\left|y(t)-y^{*}(t)\right|+1 \\
& :=K \quad \forall t>0 .
\end{aligned}
$$

Contrarily, there must exist $t_{*}>0$ such that

$$
V\left(t_{*}\right)=K, \quad V(t)<K \quad \forall t \in\left[-\tau^{+}, t_{*}\right) .
$$

Together with (45) and (47), we obtain

$$
\begin{aligned}
0 \leq & D^{-}\left(V\left(t_{*}\right)\right) \\
\leq & \left(\lambda-a_{1}^{-}\right)\left|z\left(t_{*}\right)\right| e^{\lambda t_{*}} \\
& +a_{2}^{+} e^{\lambda \tau^{+}}\left|z\left(t_{*}-\tau\left(t_{*}\right)\right)\right| e^{\lambda\left(t_{*}-\tau\left(t_{*}\right)\right)} \\
\leq & \left(\lambda-a_{1}^{-}+a_{2}^{+} e^{\lambda \tau^{+}}\right) K .
\end{aligned}
$$

Thus,

$$
0 \leq \lambda-a_{1}^{-}+a_{2}^{+} e^{\lambda \tau^{+}},
$$

which contradicts with (14). Hence, (46) holds. It follows that

$$
|z(t)|<K e^{-\lambda t} \quad \forall t>0 .
$$

This completes the proof of Theorem 5 .

Corollary 7. Suppose that all conditions in Theorem 5 are satisfied. Then (1) has a unique positive almost periodic solution which is globally exponentially stable.

Proof. From Theorems 5 and 6, we know that (4) has a unique almost periodic solution $y^{*}(t)$, which is globally exponentially stable. Consider that $x(t)$ and $y(t)$ are the solutions of (1) and (4) with the relation $x(t)=e^{y(t)}>0$ and the relevant initial conditions $\varphi$ and $\phi \in C$ satisfy $\varphi=e^{\phi} \epsilon$ $C_{+}$; it is natural to get that (1) has a unique positive almost periodic solution $x^{*}(t)$, which is also globally exponentially stable.

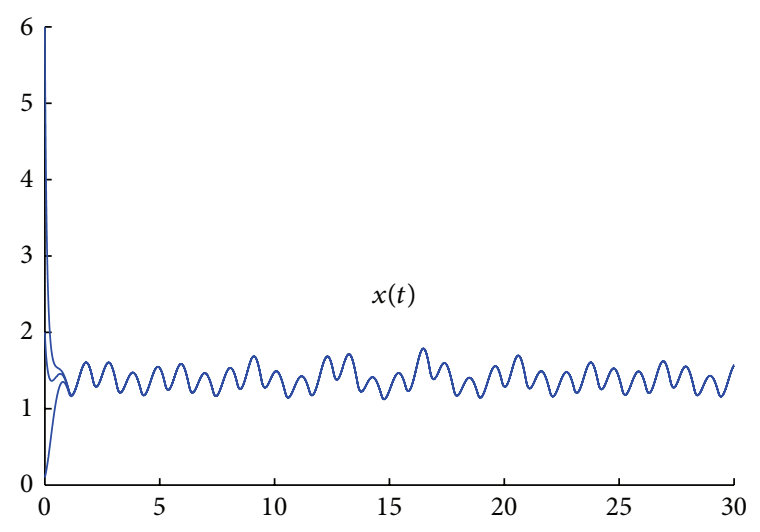

FIGURE 1: Numerical solution $x(t)$ of system (51) for initial value $\varphi(t) \equiv 0.1,2,6$.

\section{An Example}

In this section, we present an example to check the validity of our results obtained in the previous sections.

Example 1. Consider the following delay logarithmic population equation:

$$
\begin{aligned}
x^{\prime}(t)=x(t) & {[2|\sin 3 t|-(3+\cos \sqrt{3} t) \ln x(t)} \\
& \left.-\left(1+\frac{1}{2} \sin \sqrt{2} t\right) \ln x\left(t-\cos ^{2} \sqrt{5} t\right)\right] .
\end{aligned}
$$

Obviously, $\gamma(t)=2|\sin 3 t|, a_{1}(t)=3+\cos \sqrt{3} t, a_{2}(t)=1+$ $(1 / 2) \sin \sqrt{2} t, \tau(t)=\cos ^{2} \sqrt{5} t$. It is easy to get

$$
a_{1}^{-}-a_{2}^{+}=2-1.5=0.5>0,
$$

which implies that (51) satisfies the assumptions of Corollary 7. Therefore, (51) has a unique positive almost periodic solution $x^{*}(t)$, which is globally exponentially stable with the exponential convergent rate $\lambda \approx 0.15$. The numerical simulation in Figure 1 strongly supports the conclusion.

Remark 8 . Since $[14,15]$ only obtain the existence and local exponential stability of positive almost periodic solutions for delay logarithmic population model (1), one can observe that all the results in this literature and the references therein can not be applicable to prove the existence and global exponential stability of positive almost periodic solution for (51). This implies that the results of this paper are essentially new.

\section{Conflict of Interests}

The authors declare that they have no conflict of interests.

\section{Authors' Contribution}

The authors have made this paper independently. The authors read and approved the final version. 


\section{Acknowledgments}

The authors are grateful to the referees for their suggestions that improved the writing of the paper. This work was supported by the National Natural Science Foundation of China (Grant nos. 11301341 and 11201184), the Natural Scientific Research Fund of Zhejiang Provincial of China (Grant no. LY12A01018), and the Natural Scientific Research Fund of Zhejiang Provincial Education Department of China (Grant no. Z201122436). Innovation Program of Shanghai Municipal Education Commission (Grant no. 13YZ127), and Shanghai Fiscal Special Fund (Grant no. A4- 4902-13-12).

\section{References}

[1] W. Zhao, "New results of existence and stability of periodic solution for a delay multispecies Logarithmic population model," Nonlinear Analysis: Real World Applications, vol. 10, no. 1, pp. 544-553, 2009.

[2] C. Wang and J. Shi, "Periodic solution for a delay multispecies Logarithmic population model with feedback control," Applied Mathematics and Computation, vol. 193, no. 1, pp. 257-265, 2007.

[3] F. Chen, "Periodic solutions and almost periodic solutions for a delay multispecies Logarithmic population model," Applied Mathematics and Computation, vol. 171, no. 2, pp. 760-770, 2005.

[4] Q. Wang and B. Dai, "Existence of positive periodic solutions for a neutral population model with delays and impulse," Nonlinear Analysis: Theory, Methods \& Applications, vol. 69, no. 11, pp. 3919-3930, 2008.

[5] Q. Wang, Y. Wang, and B. Dai, "Existence and uniqueness of positive periodic solutions for a neutral Logarithmic population model," Applied Mathematics and Computation, vol. 213, no. 1, pp. 137-147, 2009.

[6] J. O. Alzabut and T. Abdeljawad, "Existence and global attractivity of impulsive delay logarithmic model of population dynamics," Applied Mathematics and Computation, vol. 198, pp. 463-469, 2008.

[7] Y. Luo and Z. Luo, "Existence of positive periodic solutions for neutral multi-delay Logarithmic population model," Applied Mathematics and Computation, vol. 216, no. 4, pp. 1310-1315, 2010.

[8] R. Wang and X. Zhang, "Positive periodic solution for a neutral Logarithmic population model with feedback control," Applied Mathematics and Computation, vol. 217, no. 19, pp. 7692-7702, 2011.

[9] A. M. Fink, Almost Periodic Differential Equations, vol. 377 of Lecture Notes in Mathematics, Springer, Berlin, Germany, 1974.

[10] C. Y. He, Almost Periodic Differential Equation, Higher Education Publishing House, Beijing, China, 1992, (Chinese).

[11] B. Liu, "Almost periodic solutions for a delayed Nicholson's blowflies model with a nonlinear density-dependent mortality term," Advances in Difference Equations, vol. 2014, article 72, 2014.

[12] J. Meng, "Global exponential stability of positive pseudoalmost-periodic solutions for a model of hematopoiesis," Abstract and Applied Analysis, vol. 2013, Article ID 463076, 7 pages, 2013.

[13] Q. Wang, H. Zhang, and Y. Wang, "Existence and stability of positive almost periodic solutions and periodic solutions for a Logarithmic population model," Nonlinear Analysis: Theory, Methods \& Applications, vol. 72, no. 12, pp. 4384-4389, 2010.

[14] J. O. Alzabut, G. T. Stamov, and E. Sermutlu, "On almost periodic solutions for an impulsive delay Logarithmic population model," Mathematical and Computer Modelling, vol. 51, no. 5-6, pp. 625-631, 2010.

[15] J. O. Alzabut, G. T. Stamov, and E. Sermutlu, "Positive almost periodic solutions for a delay Logarithmic population model," Mathematical and Computer Modelling, vol. 53, no. 1-2, pp. 161167, 2011.

[16] B. Yang and J. Li, "An almost periodic solution for an impulsive two-species logarithmic population model with time-varying delay," Mathematical and Computer Modelling, vol. 55, no. 7-8, pp. 1963-1968, 2012.

[17] Y. Zhao, L. Wang, and H. Zhao, "Existence and exponential stability of almost periodic solutions for a neutral multispecies Logarithmic population model," Applied Mathematics and Computation, vol. 218, no. 9, pp. 5346-5356, 2012.

[18] K. Gopalsamy, Stability and Oscillations in Delay Differential Equations of Population Dynamics, vol. 74 of Mathematics and Its Applications, Kluwer Academic Publishers, Boston, Mass, USA, 1992.

[19] J. K. Hale, Theory of Functional Differential Equations, vol. 3, Springer, New York, NY, USA, 2nd edition, 1977. 


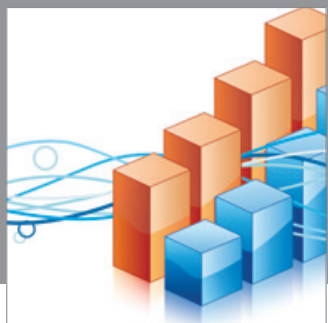

Advances in

Operations Research

mansans

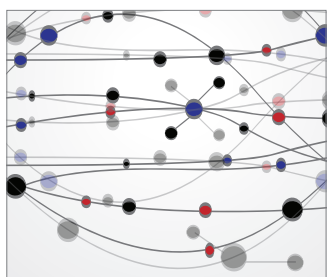

The Scientific World Journal
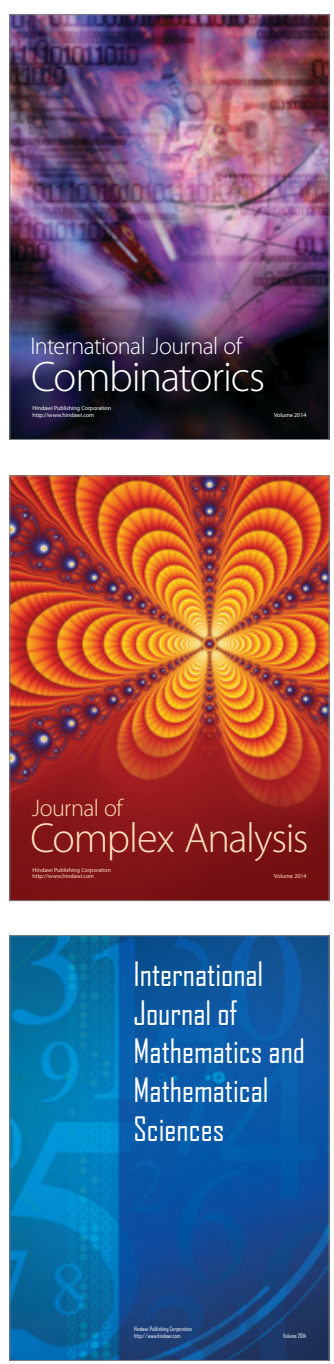
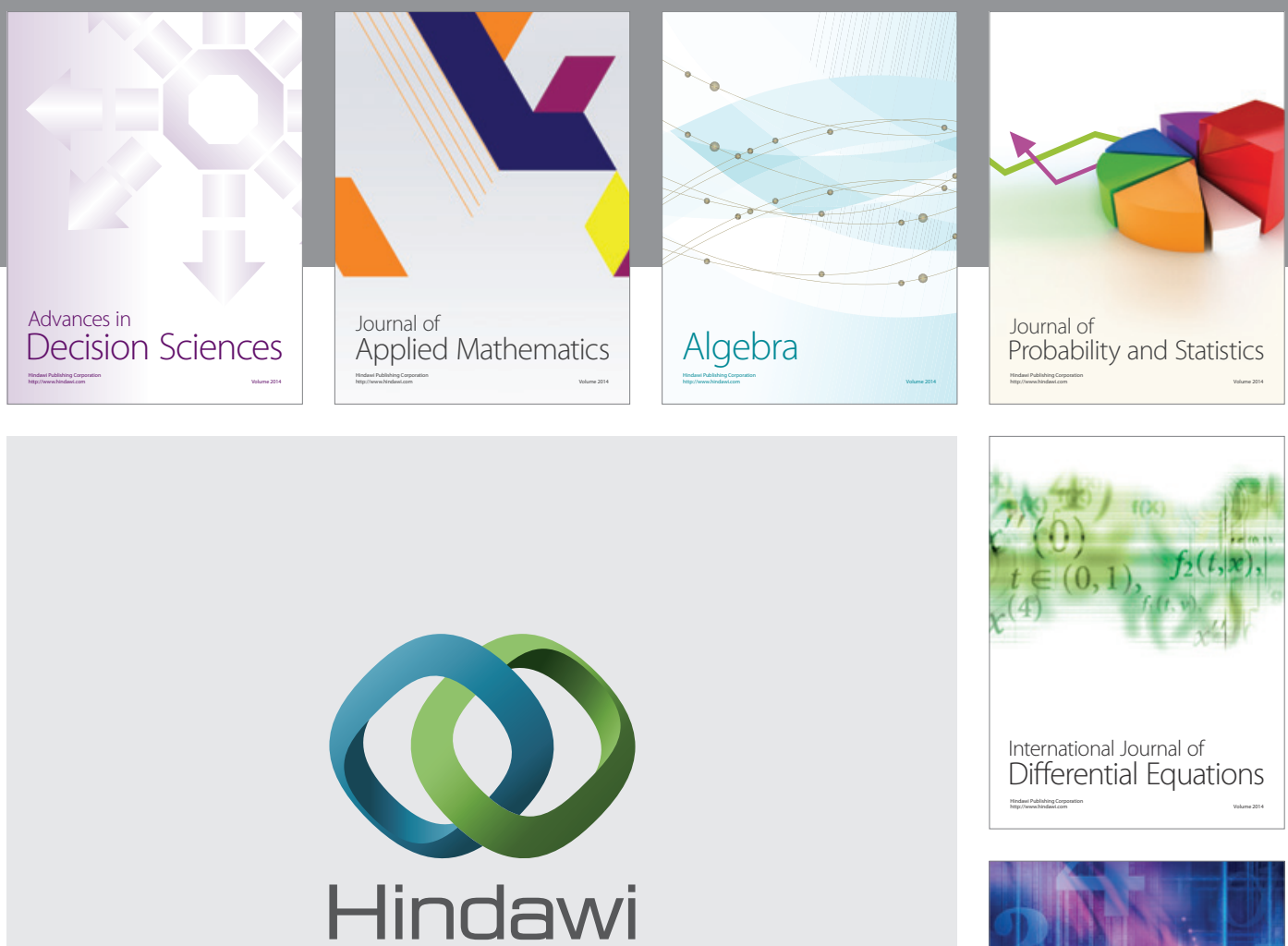

Submit your manuscripts at http://www.hindawi.com
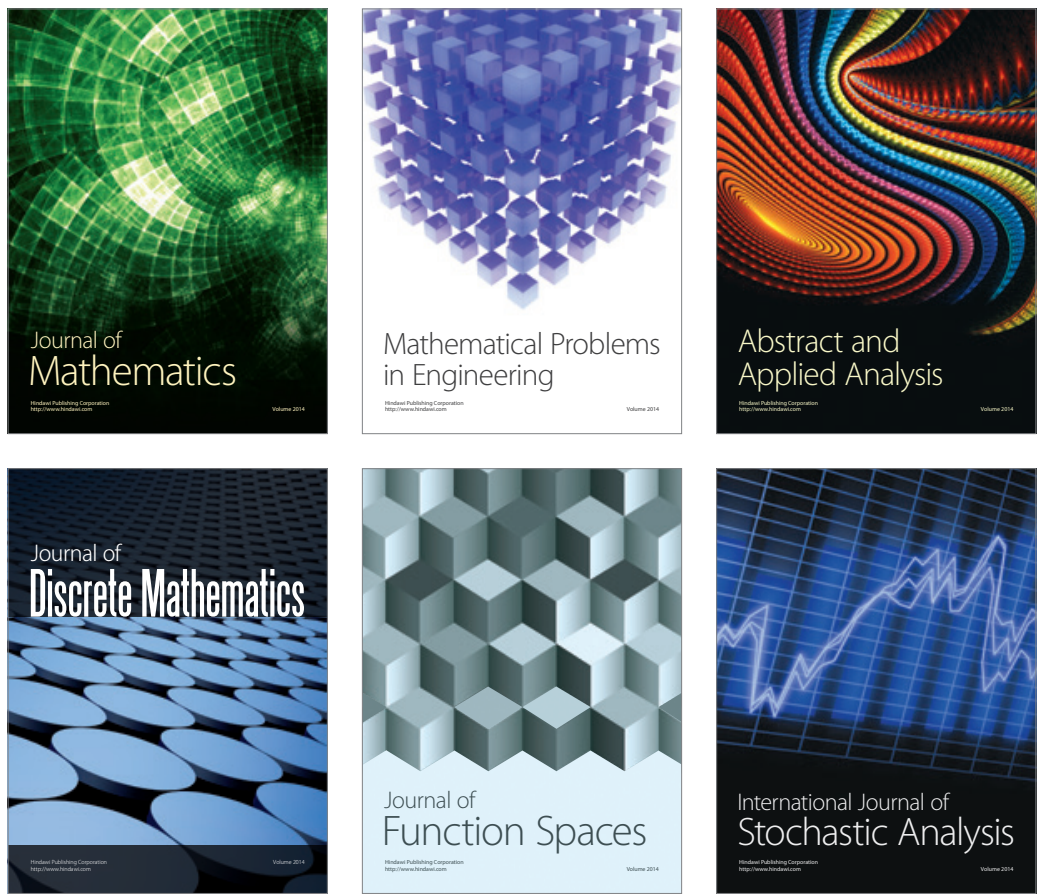

Journal of

Function Spaces

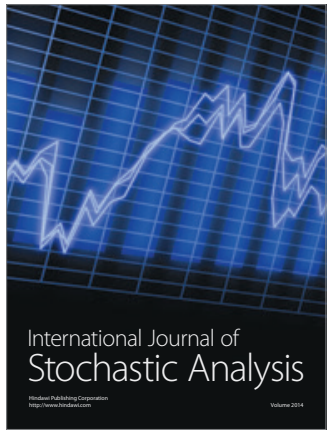

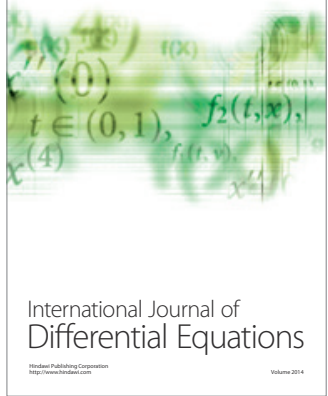
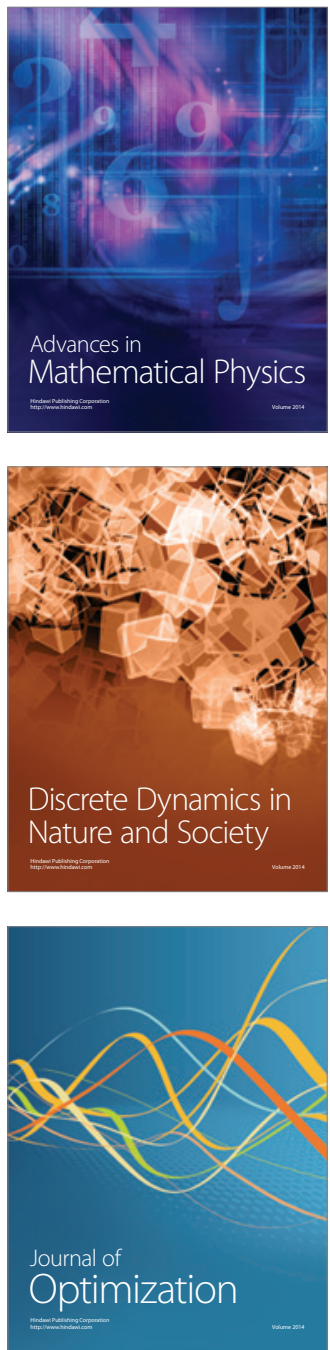